\title{
A Method of Estimating Streptomycin in Serum and Other Body Fluids by Diffusion through Agar Enclosed in Glass Tubes
}

\author{
By D. A. MITCHISON and C. C. SPICER \\ The Postgraduate Medical School of London, and Hospital for Consumption and \\ Diseases of the Chest, Brompton, London; and Imperial Cancer Research Fund, London
}

SUMMARY: Streptomycin may be estimated in body fluids by allowing the fluid to diffuse through nutrient agar seeded with a culture of Staphylococcus aureus contained in small-diameter $(3 \mathrm{~mm}$. internal) glass tubes. The zone of inhibition produced is affected neither by the anaerobic conditions within the agar nor by the volume of the test fluid. Acidity of the test fluid invalidates the results, since the depth of the zone of inhibition is decreased. Increase in the size of the inoculum of the test organism also decreases the depth of the zone of inhibition. Small errors arise from variations in timing during the setting-up of the test. The zones produced in the presence of different human sera differ slightly and to a degree similar to that found in the presence of various urines.

The available mathematical expressions suggest that the square of the depth of the zone of inhibition is linearly related to the log of the concentration of an antibiotic in the test fluid. Experimentally this relationship does not hold for low concentrations of streptomycin, probably due to the assumption of boundary conditions which cannot be defined with certainty; but it is a better approximation than the assumption of a linear relationship between the depth of the zone of inhibition (unsquared) and the log of the streptomycin concentration. A method of statistical analysis is given in which a weighted regression line is fitted to the squared values of the zones of inhibition in a manner analogous to probit analysis. The routine method may be refined without undue labour to the point where assays are accurate to within $+5 \%$.

One of the most widely used methods for estimating streptomycin in body fluids is that of Stebbins \& Robinson (1945). In this method the sensitivity to streptomycin of the test organism, a strain of Staphylococcus aureus, is increased by using $1 \%$ agar, slightly alkaline and with a low salt content. The solutions are applied to the surface of seeded plates in Oxford cylinders.

A twofold increase in the concentration of streptomycin in the fluid to be assayed produces a smaller increase in the diameter of the zone of inhibition than is produced if similar solutions of penicillin are used. Variations caused by the method of applying the solution to the seeded agar therefore diminish the accuracy of the estimation to a greater extent. When using conventional plate methods with Oxford cylinders, filter-paper disks or cups cut with a cork borer, this source of variation decreases the accuracy of the estimation considerably. Variation is also increased by failing to use plates of uniform size with flat bottoms, which are difficult to obtain at the present time. It has been shown by Hayes (1945) that the depth of agar in the plates affects the size of the zone of inhibition when estimating the concentration of penicillin in fluids.

To minimize these variations a method has been evolved in which the seeded agar is pipetted into lengths of $3 \mathrm{~mm}$. internal diameter glass tubing 
sealed at one end. The test fluid is then pipetted on to the top of the agar column thus formed. The procedure is more rapid than any of the plate methods and also allows for a greater flexibility of experimental design. It uses a minimum of apparatus and a measurement may be made on as little as 4 drops of serum. Under routine laboratory conditions nineteen out of twenty assays will lie within about $25 \%$ of the true value. Examples of an accurate assay procedure will be given where nineteen out of twenty assays would fall within the limits of $\pm \mathbf{5} \%$. Originally developed for use with serum as the test fluid, it can be used with the same accuracy for assaying streptomycin in cerebrospinal fluid, pleural, peritoneal and pericardial fluid and urine. With less accuracy it can be used for neutral or alkaline pus and for acid pus containing large amounts of streptomycin. It cannot be used for acid pus and most sputa containing small amounts of streptomycin. The fluids do not need to be sterile, nor do they need inactivation. The range is $1-1000 \mu \mathrm{g}$. streptomycin base $/ \mathrm{ml}$., with greatest accuracy in the range of $2-100 \mu \mathrm{g} . / \mathrm{ml}$.

\section{THE ASSAY TECHNIQUE}

Materials used. The test organism was Staphylococcus aureus, N.C.T.C. 7361, obtained originally from the Mayo Clinic where it is used for estimating streptomycin. The Staph. aureus Oxford $\mathbf{H}$ was also used and similar results obtained. Members of the genus Bacillus or one of the Gram-negative bacilli were not used because they were inhibited by fresh serum, but, under circumstances where this did not apply, they could be used in essentially the same manner. The Staph. aureus chosen was maintained by daily transfer in broth (peptone, $1 \%$; Lab Lemco (Oxo Ltd., London), $1 \%$; sodium chloride, $\mathbf{0 . 5} \%$ ), but it was possible to use the same broth culture incubated for $18 \mathrm{hr}$. and then kept in the refrigerator for a week.

The agar medium used in the assay was prepared from nutrient $2 \%$ agar (digest broth containing $2 \%$ Japanese agar) by diluting one volume with an equal volume of $1 \%$ peptone and adjusting the $\mathrm{pH}$ to $7 \cdot 8-8 \cdot 0$. When this agar was diluted to $0.67 \%$ and sloped in $6 \times \frac{5}{8}$ in. tubes the slopes retained their shape after $18 \mathrm{hr}$. incubation; with agar diluted to $\mathbf{0 . 4 4} \%$ they collapsed. The nutrient $1 \%$ agar was autoclaved in $19 \mathrm{ml}$. amounts in $1 \mathrm{oz}$. screw-capped bottles. Nutrient agar of the same gelling power, made with New Zealand agar, was less satisfactory because it was more difficult to pipette and the zones of inhibition were smaller.

Glass tubing of $3 \mathrm{~mm}$. internal diameter was cut into $7-8 \mathrm{~cm}$. lengths. These were sealed at one end and dry sterilized in bulk. When the tubing was narrower filling became difficult, whereas when it was wider the quantity of fluid required for the test was increased. After use the tubes were cleaned without individual handling by boiling in soap or Kinray powder solution (Reddish Chemical Works, Reddish, Stockport) followed by boiling first in dilute hydrochloric acid and then in distilled water. A microscope with a vernier millimetre scale attached to the mechanical stage and a cross-wire in the eyepiece was used for reading the depth of the inhibition zone. 


\section{Method}

(1) The nutrient $1 \%$ agar was. thoroughly melted in a screw-capped container and transferred to a $45^{\circ}$ water-bath. It was often convenient to leave it in a $56^{\circ}$ water-bath and then transfer it for a few minutes before use to a $45^{\circ}$ water-bath on the bench.

(2) The fluids to be tested were prepared and controls made up. Serum and cerebrospinal fluid needed no preparation. Urine was adjusted to $\mathrm{pH} \mathbf{7 \cdot 8}$, using cresol red in aqueous solution as an internal indicator, and then diluted 1 in 10 or 1 in 100 in $\mathrm{m} / 15$ phosphate buffer ( $\mathrm{pH} \mathrm{7.8),} \mathrm{since} \mathrm{urine} \mathrm{usually}$ contains a high streptomycin concentration. For the controls two different streptomycin concentrations were used, values being chosen so that one was at the upper limit and one at the lower limit of the expected range of the 'unknowns'. Thus in measuring the amount of streptomycin present in the serum of patients receiving $0.5 \mathrm{~g}$. streptomycin intramuscularly every $6 \mathrm{hr}$., the amounts present in the serum ranged from about 6 to $30 \mu \mathrm{g} . / \mathrm{ml}$. Controls of 4 and $32 \mu \mathrm{g} . / \mathrm{ml}$. were therefore suitable. When the range of values to be tested was unknown, controls at 4 and $64 \mu \mathrm{g} . / \mathrm{ml}$. were made up. When testing serum the controls were made up in human serum. When testing cerebrospinal fluids they were made up in distilled water. Urine standards were made up in the phosphate buffer used for dilution.

(3) A roll of plasticine (Harbutt's Plasticine Works, Bath) was placed on the bench and into it were inserted vertically groups of tubes, a group for each specimen to be tested. Each of the groups for an unknown specimen contained four tubes and each for a control six tubes.

(4) From an overnight culture of the Staph. aureus grown at $37^{\circ}, 0 \cdot 1 \mathrm{ml}$. was taken and added to $10 \mathrm{ml}$. sterile water; $1 \mathrm{ml}$. of this was added to the melted agar (final dilution of 1 in 2000). With a smaller inoculum the edge of the zone of inhibition became difficult to define, and with a larger one the sensitivity of the test was decreased.

(5) The agar was then shaken vigorously to mix in the inoculum thoroughly and, with a wide-bored Pasteur pipette the seeded agar was pipetted into all the tubes as rapidly as possible. The columns of agar in the tubes were about 2-3 $\mathrm{cm}$. long. As the pipette was withdrawn, a little agar was sucked back to ensure an evenly shaped meniscus.

(6) The agar was allowed to set for at least 5 min.; a shorter interval allows fluid to enter between the agar and the wall of the tube, causing an abnormally large zone of inhibition in a proportion of the tubes. The control and test fluids were then gently pipetted on to the tops of the agar columns. The volume added was immaterial so long as it produced a layer at least $1 \mathrm{~mm}$. deep.

(7) The tubes were incubated overnight at $37^{\circ}$ in a labelled rack. The depth of zone of inhibition was not altered by incubation for a further $48 \mathrm{hr}$.

(8) The depth of the zone of inhibition was read by using the millimetre scale on the traversing mechanical stage of a microscope. A small ridge of plasticine was put on each end of a microscope slide which was placed on the 
mechanical stage. The tubes were laid across the two ridges. The eyepiece cross-wire was focused on the centre of the meniscus between fluid and agar using a $\frac{2}{3}$ in. objective and reduced lighting. The readings on the scale were taken to the nearest $0.1 \mathrm{~mm}$. The stage was traversed till the cross-wire was at the edge of the growth. The difference between the two readings was the depth of the zone of inhibition $(y)$. As one passes towards the test fluid there is first a zone of normal growth with well-spaced colonies. There is then a zone in which the colonies are of increased size and which ends abruptly. Measurements were taken from this latter edge. Since these larger colonies are as numerous as in the zone of normal growth, and since the zone has an appreciable width, it would seem unlikely that the increased size is due solely to a diminished competition for living space. As one proceeds farther towards the test fluid, one can still see a few very small colonies; their numbers gradually decrease. The sharp definition of the edge thus appears to be due to some factor causing an increase in colony size rather than to bacteriostasis alone. The decreased size of the boundary colonies was always relatively larger when measurements were made on serum than when cerebrospinal fluid or buffer solutions were used. When acid solutions containing streptomycin in low concentrations were used the zone of growth lacked this sharp edge and, instead, the number of colonies gradually decreased over a distance of $0 \cdot 2-0.3 \mathrm{~mm}$. or more. When this occurred the inhibition zones were always smaller than with an alkaline solution of the same concentration. Penicillin, extreme degrees of acidity or alkalinity, phenol, ethanol and acetone all tended to produce zones of inhibition of the latter type, and their presence in the fluid to be assayed could thus be recognized.

(9) The measurements for each tube having been made, the average for each group of tubes was taken and this figure squared. A graph was prepared with the square of the average zone of inhibition as the ordinate and the $\log _{10}$ of the streptomycin concentration as the abscissa. The two control points on this graph were plotted and joined with a straight line. This line was used for reading off the $\log _{10}$ of the streptomycin concentration in the unknown fluid. The actual value was obtained by conversion using an antilog table. A typical example is given in Table 1.

\section{Criteria for the method and notes on sources of error}

Effect of anaerobic conditions in the agar columns. One of the first criticisms of such a method that would be made is that anaerobic conditions are established in the depths of agar columns, and that it has been reported (Geiger, Green \& Waksman, 1946; May, Voureka \& Fleming, 1947) that anaerobiosis affects the minimum concentration at which streptomycin inhibits the growth of organisms. Thus it would seem to follow that a long column of fluid above the agar column would increase the degree of anaerobiosis in the top layers of the agar and that the level at which growth occurred would be altered. This was investigated by two experiments. In the first of these (see Table 2), streptomycin was incorporated in assay agar to a concentration of 
$10 \mu \mathrm{g} . / \mathrm{ml}$. This agar was pipetted on to the top of seeded assay agar in tubes in three groups in which the length of the superposed column of agar containing streptomycin varied. There is no significant difference in the depth of the zones of inhibition caused by the three different column lengths. A similar experiment in which columns of fluid of differing length were similarly added also failed to show significant differences in the depths of the zones of inhibition.

Table 1. Example of the method and assay of streptomycin by measuring the depth of the inhibition zone in columns of inoculated agar medium

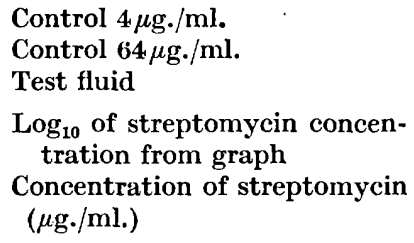

\section{Depth of zone of inhibition (mm.)}

$3 \cdot 7,3 \cdot 7,3 \cdot 4,3 \cdot 6,3 \cdot 5,3 \cdot 8$

$7 \cdot 1,7 \cdot 4,7 \cdot 1,7 \cdot 0,7 \cdot 2,7 \cdot 0$

$5 \cdot 6,5 \cdot 3,5 \cdot 4,5 \cdot 4$
Average $y^{2}$

$13 \cdot 10$

$50 \cdot 90$

$29 \cdot 43$

Table 2. The effect of anaerobic conditions on the zone of inhibition. Each group contained six tubes

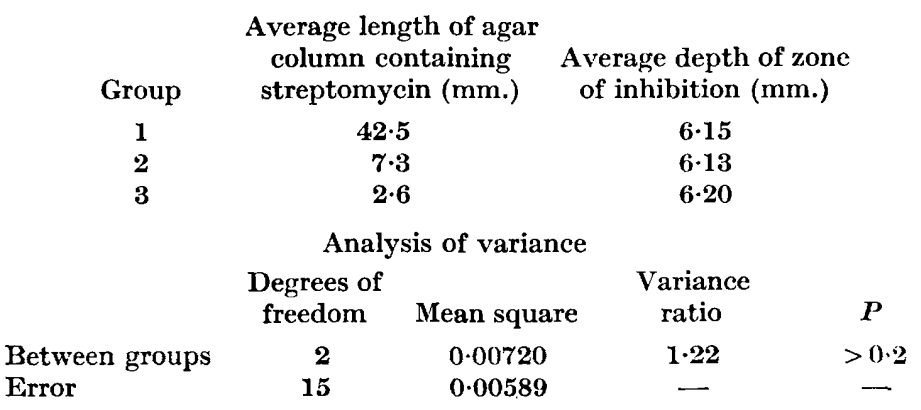

In the second experiment assay agar was seeded with Staph. aureus and then divided into a series of portions to each of which was added streptomycin to make a series of twofold dilutions from 4 to $0.03 \mu \mathrm{g} . / \mathrm{ml}$. From each, agar was pipetted into tubes to form column lengths of about $45 \mathrm{~mm}$. These were incubated for $18 \mathrm{hr}$. at $37^{\circ}$. In the tubes containing $0.06 \mu \mathrm{g}$. streptomycin $/ \mathrm{ml}$. or less there were numerous colonies throughout the column. In the tube containing $0 \cdot 125 \mu \mathrm{g}$. streptomycin $/ \mathrm{ml}$. and in those with higher concentrations there were very scanty colonies. In all tubes the number of colonies was approximately even throughout the column. Thus the end-point at which the majority of the cocci failed to grow was not altered by the change from aerobic to anaerobic growth that occurred over the length of the agar column.

Effect of the $\mathrm{pH}$ of the fluid to be assayed. Waksman, Bugie \& Schatz (1944) and others have reported that the activity of streptomycin is diminished in acid solutions. It might thus be expected that the zones of inhibition would be diminished when acid fluids containing low concentrations of streptomycin are assayed. To investigate this, concentrations of streptomycin of 5 and $1 \mu \mathrm{g} . / \mathrm{ml}$. 
were made up in $\mathrm{M} / \mathbf{1 5}$ phosphate buffer at a range of $\mathrm{pH}$ values from $5 \cdot 8$ to 10 , and concentrations of $100 \mu \mathrm{g}$. streptomycin $/ \mathrm{ml}$. were made up in $\mathrm{M} / 5$ phosphate buffer over the same range. The results are plotted in Fig. 1 . It is clear that all fluids to be assayed should thus have a $\mathrm{pH}$ value of $\mathbf{7 \cdot 8}$ or more. This is of importance in measuring streptomycin in urine, pus or sputum, any of which may be acid.

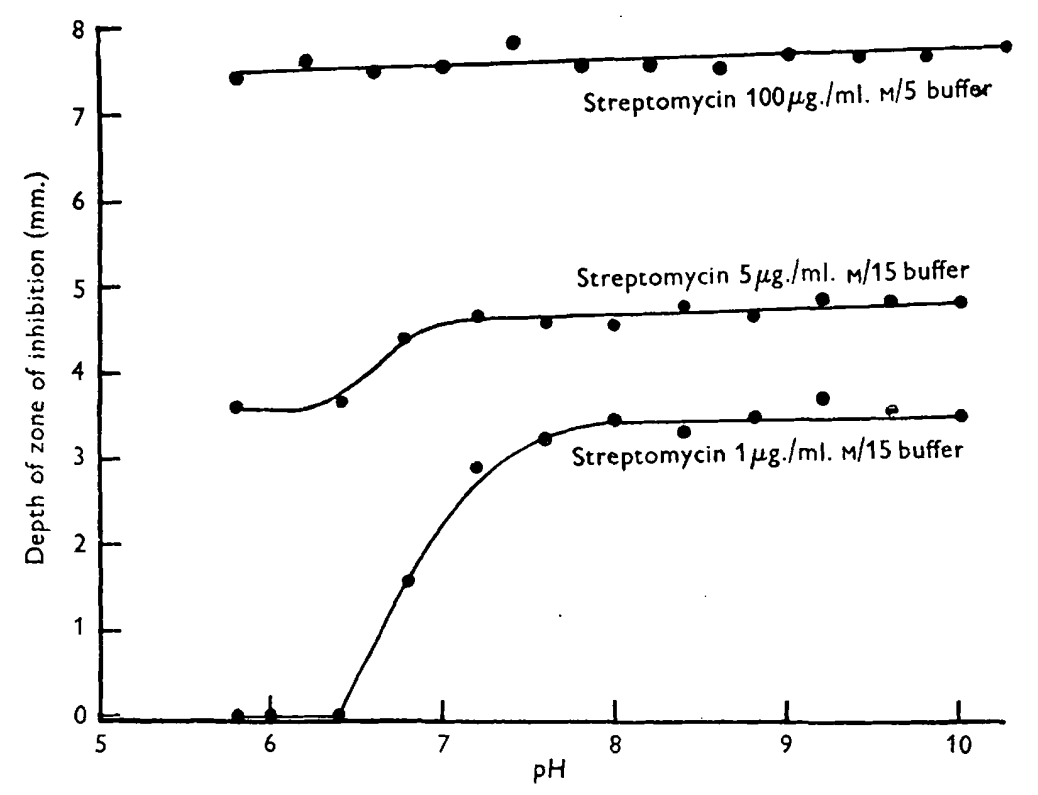

Fig. 1. Effect of $\mathrm{pH}$ on the zone of inhibition.

Effect of inoculum. An $18 \mathrm{hr}$. growth of Staph. aureus was added to batches of assay agar to make final dilutions of $1 / 4000,1 / 2000,1 / 1000,1 / 500,1 / 250$, and from each batch tubes were set up. $\mathrm{m} / 10$ phosphate buffer ( $\mathrm{pH} 7.8$ ) containing 4, 40 and $200 \mu \mathrm{g}$. streptomycin $/ \mathrm{ml}$. was added above the agar columns. The timing of the setting up of the different batches was kept as similar as possible. The results are shown in Fig. 2 . It will be scen that the smaller the inoculum, the steeper is the slope of the line relating length of zone of inhibition to $\log _{10}$ concentration of streptomycin. 'Thus, to increase the sensitivity of the method, the inoculum should be as small as is consistent with accurate determination of the edge of the zone of growth.

Standard curves prepared from different tubes of seeded agar even under apparently identical conditions were found to differ from each other both in height and slope, sometimes very markedly. Probably a considerable part of this variation was due to small alterations in the size of the inoculum though there might also have been other factors. 'The tubes for controls and unknowns to be tested against them were always set up from the same tube of agar. Breach of this rule introduced very large errors.

Errors in timing. When a row of tubes was filled there was a difference between the tubes inasmuch as the first tube was filled a few minutes before 
the last. This introduced a further possible source of error. If the processes of filling with agar and adding the fluid to be assayed were carried out as rapidly as possible the errors involved were very small and could be ignored in routine examinations. Filling with agar was completed in 2-3 min. or less, and adding the fluid to be assayed in $10 \mathrm{~min}$. or less. For more accurate work the effect

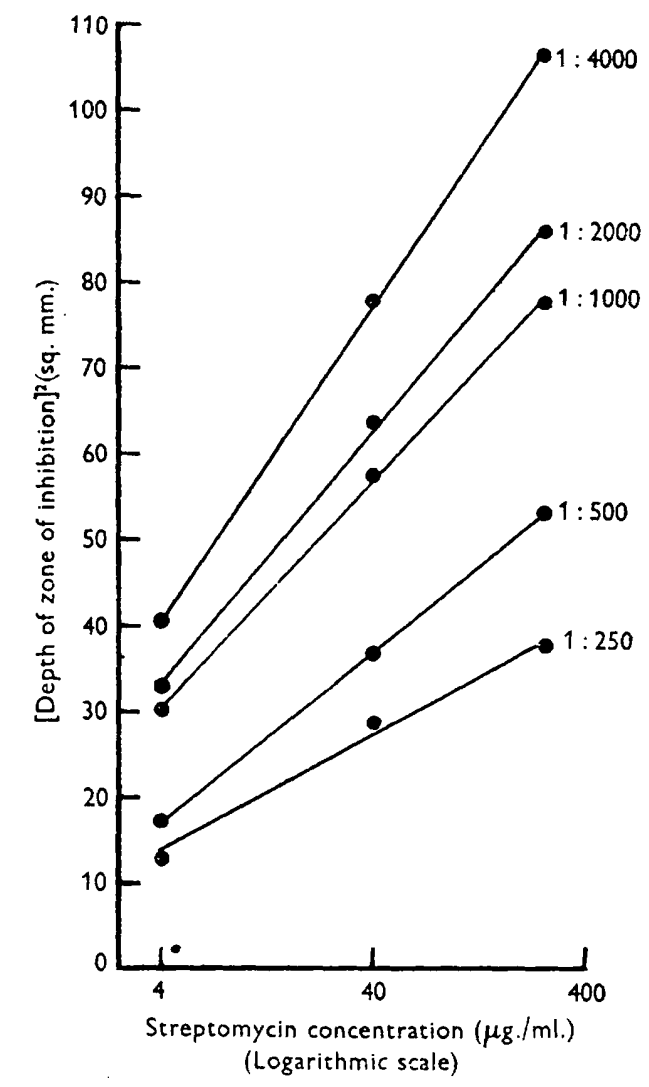

Fig. 2. Effect of inoculum on the zone of inhibition. Final dilutions of an $18 \mathrm{hr}$. broth culture from $1: 250$ to $1: 4000$.

of the timing errors could not be ignored. It was investigated in conjunction with the construction of curves relating zone of inhibition to concentration of streptomycin in the fluid to be assayed.

In considering the timing errors there were two possible sources of uncontrolled variation. One of these was in the adding of the agar to the tubes and the other was in the adding of the fluid above the agar. Restraints could be applied to these two variables and, at the same time, the relation between zone of inhibition and streptomycin concentration could be investigated by the use of a Latin square experiment. A typical example is given in Table 3. Agar was added to the tubes in row 1 first, then row 2 , etc. The fluids were added above the agar in column 1 first, then in column 2, etc. The total time taken 
to add the agar was $5 \mathrm{~min}$. and to add the fluid $12.5 \mathrm{~min}$. There were two tubes in each 'plot'. In this case streptomycin was made up in $\mathrm{m} / \mathbf{1 5}$ phosphate buffer $(\mathrm{pH} 7 \cdot 8)$ in concentrations of $256,64,16,4$ and $1 \mu \mathrm{g} . / \mathrm{ml}$. The concentration used is indicated in the corner of each plot.

Table 3. Latin square experiment; the effect of timing errors and different concentrations of streptomycin on the depth $(\mathrm{mm}$.) of the zone of inhibition

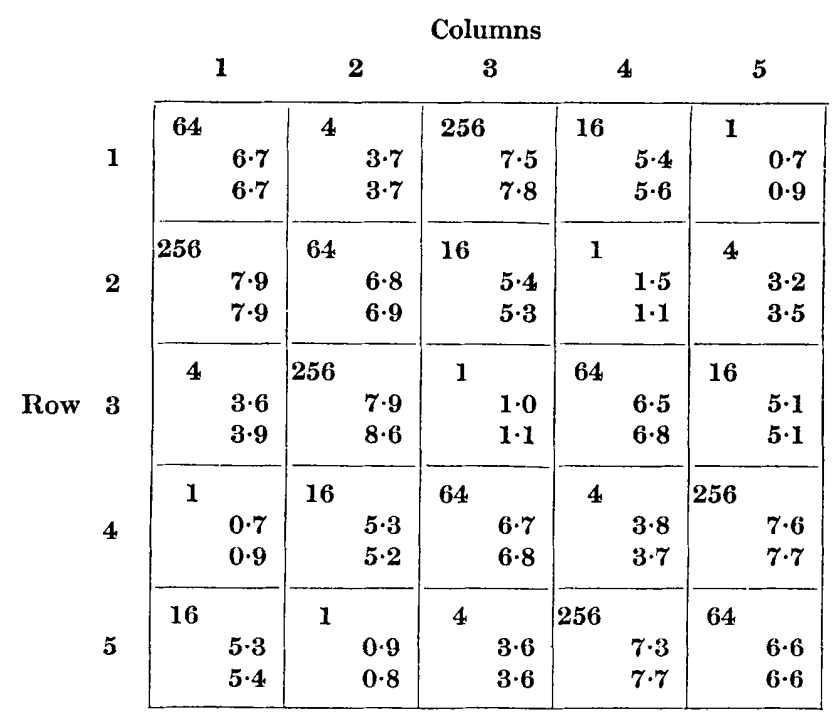

Concentration of streptomycin $(\mu \mathrm{g} . / \mathrm{ml}$ ) in top left corners.

Table 4. Effect of timing errors in six Latin square experiments

\begin{tabular}{|c|c|c|c|c|c|}
\hline \multirow{2}{*}{$\begin{array}{l}\text { Latin } \\
\text { square }\end{array}$} & \multirow{2}{*}{$\begin{array}{c}\text { Range of } \\
\text { streptomycin } \\
\text { concentrations } \\
(\mu \mathrm{g} . / \mathrm{ml} .)\end{array}$} & \multicolumn{2}{|c|}{$\begin{array}{c}\begin{array}{c}\text { Approximate time (min.) } \\
\text { taken in }\end{array} \\
.\end{array}$} & \multicolumn{2}{|c|}{$\begin{array}{l}\text { Probability } \\
\text { of occurrence by chance } \\
\text { Variability due to }\end{array}$} \\
\hline & & Adding agar & Adding fluid & Adding agar & Adding fluid \\
\hline $6 \times 6$ & $2-512$ & 6 & 18 & $0.05-0.01$ & $0 \cdot 2-0 \cdot 05$ \\
\hline $5 \times 5$ & $1-256$ & 5 & $12 \cdot 5$ & $>0.2$ & $0.01-0.09$ \\
\hline $4 \times 4$ & $4-5$ & 4 & 8 & $0 \cdot 05-0 \cdot 01$ & $>0 \cdot 2$ \\
\hline $6 \times 6$ & $3-5$ & 6 & 18 & $>0 \cdot 2$ & $0.2-0.05$ \\
\hline $6 \times 6$ & $3-5$ & 6 & 18 & $0.01-0.001$ & $0 \cdot 05-0.01$ \\
\hline $12 \times 12$ & $0 \cdot 8-100$ & 15 & 40 & $<0.001$ & $<0.001$ \\
\hline
\end{tabular}

From the analysis of variance (see below), it was concluded that the timing error, due to the adding of the agar, was of no significance $(P=0 \cdot 2)$, whereas that due to the addition of the fluid above the agar was significant $(P=0 \cdot 05-0 \cdot 01)$. An analysis was made of two similar experiments, one with streptomycin in $\mathrm{M} / 15$ buffer ( $\mathrm{pH} \mathrm{7.8)}$ and the other with streptomycin in serum. Three similar lay-outs were used for two point assays, one a $4 \times 4$ Latin square, the other two being $6 \times 6$ squares. A sixth experiment using a $12 \times 12$ Latin square was more complicated and involved longer times in setting up. The probabilities that the timing errors would occur by chance are given in Table 4 . It thus 
becomes apparent that both timing errors, though small, were usually significant. The relation between the depth of the zone of inhibition and the streptomycin concentration in the fluid to be assayed was also investigated in the experiment given in Table 3. It is discussed theoretically in the appendix together with a statistical analysis of the experimental results.

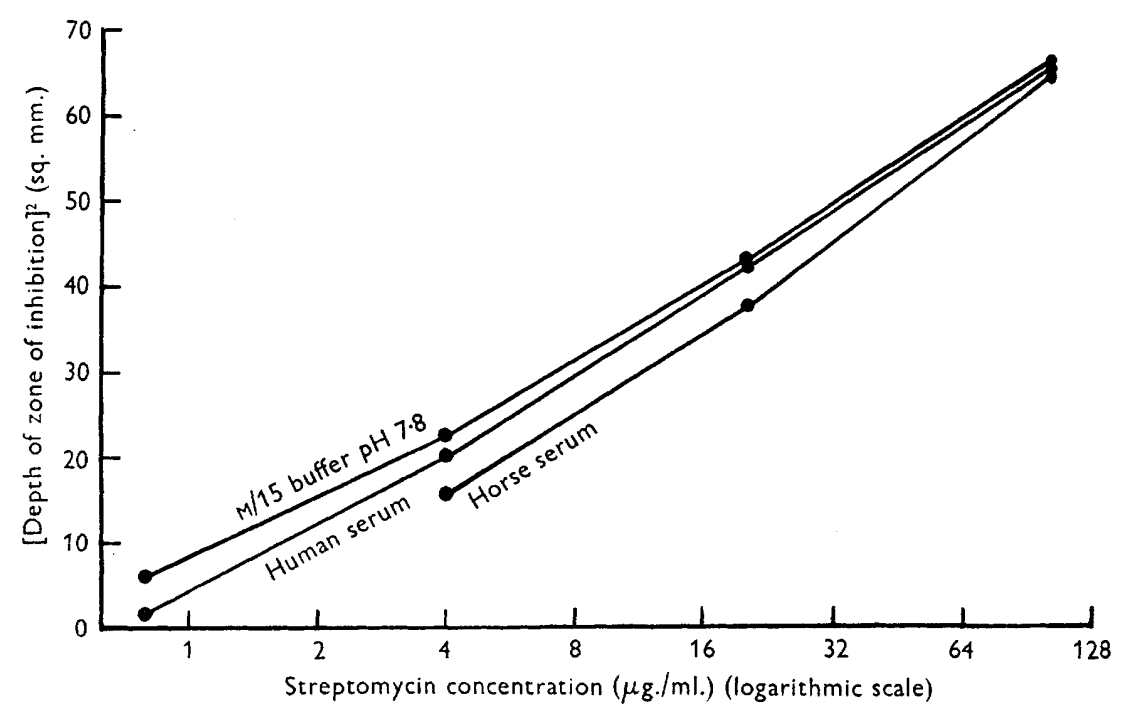

Fig. 3. Curves relating the zone of inhibition to the streptomycin concentration in the assay fluid. Comparison between $\mathrm{M} / \mathbf{1 5}$-phosphate buffer $\mathrm{pH} \boldsymbol{7 \cdot 8}$, human serum and horse serum.

The influence of the fluid containing streptomycin. The necessity of using human serum for the controls when estimating serum streptomycin was investigated by making up four concentrations of streptomycin in pooled human serum, in horse serum and in $\mathrm{M} / 15$ phosphate buffer $(\mathrm{pH} 7 \cdot 8$ ) and comparing them in a $12 \times 12$ Latin square, using the rows and columns to restrain the two timing errors. The buffer solution gave a curve (Fig. 3) closer to the one derived from the horse serum. Even so there was a highly significant difference between the slope and the height of the curves for human serum and buffer. (Between means $t=44 \cdot 8$; between regressions $t=9 \cdot 39$. For both $\boldsymbol{P}<0.001$.)

To determine whether all human sera containing the same concentrations of streptomycin give the same zones of inhibition streptomycin was added to twenty different sera to make a final concentration of $10 \mu \mathrm{g} . / \mathrm{ml}$. Each serum was added to six tubes. The tubes were arranged in two sets of fifteen balanced incomplete blocks in which both timing errors were incorporated in the interblock differences. The average zones of inhibition produced after adjustment to remove the interblock differences are shown in Table 5. As will be seen from the analysis of variance the differences between the zones produced by different sera were small but real. They should be taken into account in estimating the 
error of the methods when the control serum is different from the test serum. The mean square for 'between sera' was approximately three times the error mean square. The error of $x_{3}$, the unknown serum derived from equation (15) in the appendix, should therefore be multiplied by $\sqrt{3}$ or 1.73 . An analysis on similar lines for urines indicated an almost identical amount of variation.

Table 5. Zones of inhibition with twenty different sera

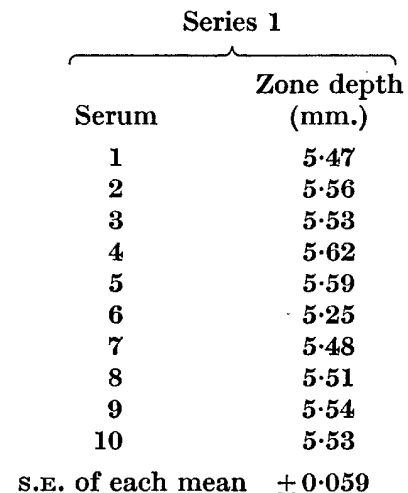

$\begin{array}{cc}\text { Serum } & \begin{array}{c}\text { Zone depth } \\ (\mathrm{mm})\end{array} \\ 11 & 5 \cdot 12 \\ 12 & 5 \cdot 00 \\ 13 & 5 \cdot 00 \\ 14 & 4 \cdot 97 \\ 15 & 5 \cdot 01 \\ 16 & 5 \cdot 05 \\ 17 & 4 \cdot 86 \\ 18 & 5 \cdot 09 \\ 19 & 4 \cdot 96 \\ 20 & 4 \cdot 96 \\ & \pm 0 \cdot 042\end{array}$

\begin{tabular}{lcccc} 
& \multicolumn{2}{c}{ Analysis of variance } \\
& $\begin{array}{c}\text { Degrees of } \\
\text { freedom }\end{array}$ & $\begin{array}{c}\text { Mean } \\
\text { square }\end{array}$ & $\begin{array}{c}\text { Variance } \\
\text { ratio }\end{array}$ & $\boldsymbol{P}$ \\
Series 1: & & & & \\
Between blocks & 14 & $0 \cdot 03852$ & $2 \cdot 23$ & $0 \cdot 05-0.01$ \\
Between sera & 9 & $0 \cdot 05331$ & $3 \cdot 08$ & $0 \cdot 05-0 \cdot 01$ \\
Error & 36 & $0 \cdot 0173$ & - & - \\
Series 2: & & & & \\
Between blocks & 14 & $0 \cdot 01786$ & $2 \cdot 019$ & 0.02 \\
Between sera & 9 & $0 \cdot 02796$ & $3 \cdot 161$ & $0 \cdot 01$ \\
Error & 36 & $0 \cdot 00884$ & - & -
\end{tabular}

Notes on an accurate assay procedure

The fluid containing the unknown concentration of streptomycin was assayed approximately so that its value was known within $\pm 25 \%$ of the true figure. The standard was made up in a concentration of $4 \mu \mathrm{g}$. streptomycin $/ \mathrm{ml}$. The unknown was adjusted to as near $4 \mu \mathrm{g} . / \mathrm{ml}$. as possible, and three parts of both the standard and unknown solution were diluted each with one part of the diluting fluid. These four solutions were then compared in a $4 \times 4$ Latin square, in which the two timing errors were incorporated in the rows and columns. Each plot in the square contained two tubes so that eight tubes were used for each solution. Each reading was squared and the averages of these figures were taken. Let the zones produced by the standard solutions be $y_{11}$ and $y_{12}$ and the zones produced by the unknown $y_{21}$ and $y_{22}$. Let $d$ be the difference between the $\log _{10}$ concentrations of the two standard and unknown 
solutions, i.e. $\mathbf{0} \cdot \mathbf{1 2 4 9 4}$, and let $M$ be the difference between the $\log _{10}$ concentrations of standard and unknown. Then (Finney, 1944),

$$
\begin{array}{ll}
R=\frac{1}{2}\left(y_{22}-y_{21}+y_{12}-y_{11}\right), & T=\frac{1}{2}\left(y_{22}-y_{21}-y_{12}+y_{11}\right), \\
S=\frac{1}{2}\left(y_{22}+y_{21}-y_{12}-y_{11}\right), & M=-S d / R .
\end{array}
$$

Standard error of

$$
M= \pm \frac{s d}{R^{2}} \sqrt{\frac{R^{2}+S^{2}}{n}}
$$

$T$ is a test of parallelism and its standard error is $s / \sqrt{ } n ; n=$ the number of tubes used for each concentration, i.e. $8 ; s^{2}=$ the error term derived from the analysis of variance of the squared figures. Since the range of the response is small this

\begin{tabular}{|c|c|c|c|c|}
\hline Assay & $\begin{array}{c}\text { Estimated streptomycin } \\
\text { concentration } \\
(\mu \mathrm{g} . / \mathrm{ml} .)\end{array}$ & $\begin{array}{c}\text { True streptomycin } \\
\text { concentration } \\
(\mu \mathrm{g} . / \mathrm{ml} .)\end{array}$ & $\begin{array}{l}\text { Actual } \\
\text { error } \\
(\%)\end{array}$ & $\begin{array}{c}\text { Estimated error } \\
2 \times \text { s.E. of mean } \\
(\%)\end{array}$ \\
\hline 1 & $3 \cdot 445$ & 3.466 & $-0 \cdot 6$ & $\begin{array}{l}101 \\
\pm 5\end{array}$ \\
\hline 2 & $4 \cdot 264$ & $4 \cdot 231$ & +0.8 & \pm 5 \\
\hline 3 & $3 \cdot 744$ & 3.730 & +0.4 & \pm 4 \\
\hline 4 & $4 \cdot 121$ & $4 \cdot 106$ & +0.4 & \pm 4 \\
\hline
\end{tabular}
is a reasonable approximation.

Table 6. Results of four accurate assays

Four assays on these lines using known quantities of streptomycin dissolved in $\mathbf{M} / \mathbf{1 5}$ phosphate buffer $(\mathrm{pH} \mathrm{7 \cdot 8)}$ were done. Sixteen tubes for each solution were used instead of eight. The results are given in Table 6 together with the estimated and actual errors. In each case the unknown and standard lines were parallel within the limits of experimental error. The estimated error was between \pm 4 and $5 \%$, and the actual error was well within these limits. More complex arrangements for performing a number of assays simultaneously are clearly possible and would slightly increase the accuracy of the procedure.

\section{DISCUSSION}

Conventional pour-plate methods involve using numerous controls. Large volumes of agar must be melted and distributed accurately into uniform plates. All the fluids to be assayed must be accurately measured at each application. All procedures need careful timing. The results must be read to at least the nearest $\mathbf{0 . 2 5} \mathrm{mm}$., which is difficult on a plate and may entail a subjective error. All these features require such an expenditure of time and material as to make them unsuitable as a routine procedure. By the use of tubes, which are easily set up, require a small volume of agar and can be read with no subjective error, it is hoped that these difficulties have been overcome. Further, the volume of fluid required for an assay has been considerably decreased. Such a procedure provides a reasonably accurate, reliable and practicable routine method of assaying streptomycin and has been used over a period of a year for many hundreds of estimations on various fluids.

We have found it difficult to make any comparison of the accuracy of this method with other agar diffusion methods (Loo, McGuire, Savage, Skell, 
Thornberry, Erlich \& Sylvester, 1945; Kornegay, Forgacs \& Henley, 1946; Challinor \& King, 1947), since in these insufficient data are given. However, in the method described by Brownlee, Delves, Dorman, Green, Grenfell, Johnson \& Smith (1948), where a Bacillus subtilis spore suspension is used, the fiducial limits $(P=0.05)$ calculated for an assay performed under very similar conditions to those described for our accurate assay procedure are $\pm 4 \%$. Clearly the two methods are very similar in their accuracy. We would, however, claim that ours is a far simpler procedure, is more flexible and requires less apparatus.

The streptomycin used in these experiments was the hydrochloride, Lot No. 594, manufactured by Merck and Co., Rahway, New Jersey, U.S.A., and was supplied by the Streptomycin in Tuberculosis Trials Committee of the Medical Research Council. References to the weight of streptomycin used indicate the weight of the base.

\section{APPENDIX}

The relation between depth of zone of inhibition and streptomycin concentration

Results from the experiment detailed in Table 3 were used to investigate the relation between the depth of the zone of inhibition and the streptomycin content of the fluid being assayed. The average of the readings was taken for each streptomycin concentration. At the same time each of the readings was squared and the average of these taken for each streptomycin concentration. The averages for both the squared and the unsquared values have been plotted against $\log _{10}$ streptomycin concentration in Fig. 4. Before analysing these results in detail certain theoretical aspects may be considered.

Most processes of linear diffusion can be described by Fick's well-known differential equations:

$$
\frac{\partial C}{\partial T}=D \frac{\partial^{2} c}{\partial x^{2}},
$$

where particles in a concentration of $c$ diffuse over a distance $x$ in time $T$.

In considering the diffusion of streptomycin under the experimental conditions described it is found that there is a series of different solutions of this equation according to the boundary conditions chosen. Some of these boundary conditions are known while others are doubtful. The conditions assumed are as follows:

(1) That a process of diffusion occurs in the test fluid as well as in the agar column. This is likely, since the diameter of the tube is small and the tube is incubated at a constant temperature.

(2) That the two columns can each be considered as of infinite length. Experimentally it has been shown that the length of the column of the test fluid (as long as it is greater than about $1 \mathrm{~mm}$.) does not affect the depth of the zone of inhibition. In similar experiments the length of the agar column has also been shown to be immaterial. 
(3) That the rate of diffusion is the same in the test fluid as it is in the agar column. This is not, in fact, true, but the rates are probably very similar since the agar is only a $1 \%$ gel.

(4) That there is no diffusion potential due to the charge of the streptomycin ion. Lenher \& Smith (1936) have shown that no potential gradient arises when

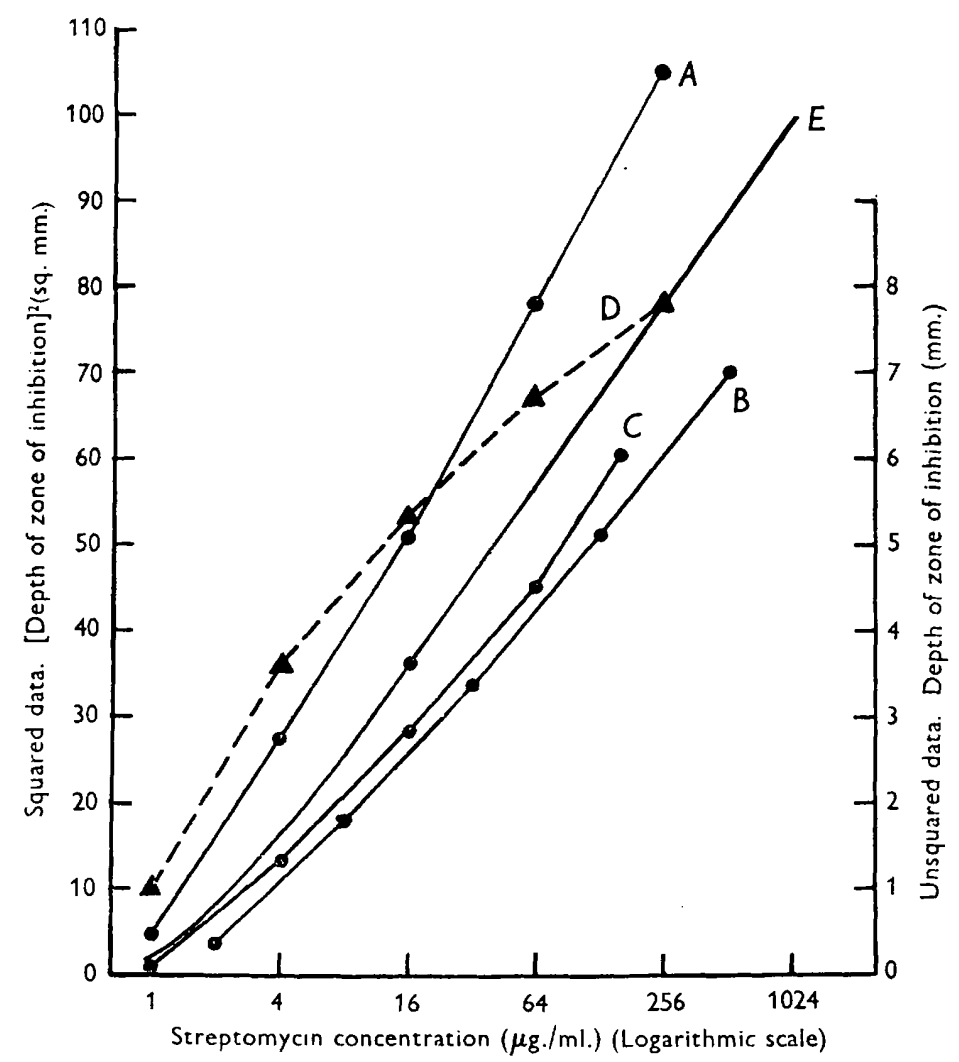

Fig. 4. Curves relating the zone of inhibition to the streptomycin concentration in the assay fluid. A, streptomycin made up in $\mathbf{M} / \mathbf{1 5}$ phosphate buffer $\mathrm{pH} 7 \cdot 8$. Squared data. B, streptomycin made up in pooled human serum. Squared data. C, streptomycin made up in $\mathrm{m} / 15$ phosphate buffer $\mathrm{pH} 7 \cdot 8$. Results of Latin square experiment detailed in Table 3. Squared data. D, the same results as in $\mathrm{C}$ but the data are not squared. $\mathrm{E}$, calculated curve from equation (2) substituting $C=0 \cdot 35$ and $2 \sqrt{ } /(D T)=4 \cdot 15$.

charged dyes diffuse into water in the presence of a few equivalents of sodium chloride. We can therefore assume that the streptomycin ion behaves like an uncharged molecule.

(5) That the composition of the seeded agar is the same throughout its depth. The concentration at which streptomycin inhibits the growth of the test organism is, in part, determined by the composition of the medium in which growth occurs (May, Voureka \& Fleming, 1947). Constituents of serum or other body fluid, of which the streptomycin content is being determined, will diffuse into the agar column and establish a gradient. The composition of the 
column must therefore vary throughout its length. However, if serum and buffer solution at $\mathrm{pH} 7 \cdot 8$ are used as test fluids, the curves relating the concentration of streptomycin that they contain to the depth of the zone of inhibition differ in height and slope but still have the same shape (Fig. 4). We have no means of estimating the effect of an osmotic gradient between the test fluid and the agar.

On the basis of these assumptions the relation between depth of zone of inhibition $y$, and concentration of streptomycin in the test fluid $C_{0}$, can be expressed (Mellor, 1912) by

$$
C=\frac{C_{0}}{2}\left[1-\Phi\left(\frac{y}{2 \sqrt{ }(D T)}\right)\right]
$$

where

$$
\Phi=\frac{2}{\sqrt{ } \pi} \int_{0}^{y} e^{-y^{2}} d x
$$

$C=$ concentration of streptomycin necessary to inhibit the test organism, $T=$ the time between adding the test fluid and the formation of the zone, $D=$ the diffusion coefficient of streptomycin defined in (1). Cooper \& Woodman (1946), in discussing the diffusion of antiseptics through agar gels, have used the expression

$$
C=C_{0} e^{-y^{2 / 4 D T}} \text {. }
$$

This expression assumes that the concentration of the antiseptic in the test fluid remains constant.

Vesterdal (1947), in considering radial diffusion of penicillin, used the expression

$$
C=\frac{A C_{0}}{\sqrt{ }(\pi D T)} e^{-y^{2} / 4 D T},
$$

where $A$ is the cross-sectioned area of the Oxford cylinder. This expression assumes that the total quantity of the penicillin is initially deposited in an infinitely thin layer on the surface of the agar.

The fundamental resemblance between equation (2) and equations (3) and (4) can be brought out using the well-known series for $\Phi(\theta)$ (Levy \& Roth, 1936)

$$
\Phi(\theta)=1-\frac{e^{-\theta^{2}}}{\theta \sqrt{ } \pi}\left(1-\frac{1}{2 \theta^{2}}+\frac{1.3}{\left(2 \theta^{2}\right)^{2}}-\frac{1.3 .5}{\left(2 \theta^{2}\right)^{3}}+\ldots\right)
$$

If $\theta$ is fairly large so that we may neglect terms of order $\theta^{-2}$, then

$$
\Phi(\theta) \bumpeq 1-\frac{e^{-\theta^{2}}}{\theta} \sqrt{\pi},
$$

so that, from (2), putting $\theta=\frac{y}{2 \sqrt{(D T)}}$, we have, on rearranging,

$$
\log C_{0}=\log (2 C \sqrt{ } \pi)+\log \left(\frac{y}{2 \sqrt{ }(D T)}\right)+\frac{y^{2}}{4 D T},
$$

when $y$ is large $\log \left(\frac{y}{2 \sqrt{ }(D T)}\right)$ becomes negligible in comparison with $\frac{y^{2}}{4 D T}$, and an approximately linear relation exists between $\log C_{0}$ and $y^{2}$.

When $y$ is small this resemblance breaks down; for we have (Levy \& Roth, 1936)

$$
\Phi(\theta)=\frac{2}{\sqrt{ } \pi}\left(\theta-\frac{\theta^{3}}{1 ! 3}+\frac{\theta^{5}}{2 ! 5}-\ldots\right),
$$


whence, ignoring terms in $y^{2}$, using (2) and rearranging,

$$
\log C_{0}=\log (2 C)+\frac{y}{\sqrt{ }(\pi D T)},
$$

so that $\log C_{0}$ is linearly related to $y$, not to $y^{2}$. It is doubtful whether any of the proposed formulae hold for small values of $y$, and from the preceding discussion it seems dangerous to deduce values for $D$ from the constants of fitted lines.

Since it is not possible to define the boundary conditions with certainty it is clearly unwise to say that these relationships hold accurately. We hope to show from experimental results that

(1) there is a close approximation to a linear relationship between $y^{2}$ and $\log C_{0}$,

(2) there is, however, a definite deviation from linearity at low concentrations of streptomycin.

These conclusions would indicate that equation (2) is a better solution than (3) or (4), since both of the latter equations stipulate a strictly linear relation between $y^{2}$ and $\log C_{0}$. It would be difficult to fit experimental values to equation (2), but, by substituting values of 0.35 for $C$ and $4 \cdot 15$ for $2 \sqrt{ }(D T)$, a curve can be drawn in the same range as two experimental curves (Fig. 4), and it is clear that it has a similar shape. We will now try to show that the results of the Latin square experiment of Table 3 yield these results.

The use of the squared data complicates the analysis somewhat, since the variance of the squared values is related closely to their mean at any given dose level, which invalidates the usual tests of significance. Under these circumstances a system of weighting must be used for deriving the error of the estimates. If one was using the unsquared data, the analysis of variance is as in Table 7. From this it is evident that the unsquared data depart significantly from linearity. The test for linearity using the squared figures has to be approached in a somewhat different manner, since the mean responses at each concentration level are of unequal accuracy and a weighted regression must be fitted. The method of doing this follows closely that worked out for probit analysis, of which several good accounts have been published (Finney, 1947 a, $b$ ). If in the terminology of probit analysis we write 'response' for 'probit', then the following relationships hold:

(1) $Y=$ response (squared reading from provisional line),

(2) $Y_{M}=Y-2 Y$ (minimum working response),

(3) $R=2 \sqrt{ } Y$ (range),

(4) $u=$ unsquared observed mean response,

(5) $Y_{W}=Y-2 Y+2 u \sqrt{ } Y$ (working response) $=Y-2 \sqrt{ } Y(\sqrt{ } Y-u)$,

(6) $W=\frac{1}{4} Y$ (weight)

The procedure is then to draw a line by eye to fit the squared responses (mean of the squared readings). From this the provisional values, $\boldsymbol{Y}$, are taken and the working responses calculated. A weighted regression of $Y_{w}$ on $C_{0}$ is then fitted in the usual manner using weights $n w$, where $n$ is the number of tubes at each concentration level. If necessary a second approximation can be 
carried out calculating the $Y^{\prime}{ }_{w}$ values from the $Y^{\prime}$ of the first line. The procedure is set out in Table 8 .

The test of linearity now consists of comparing the sum of squares of the deviations of the observed point from the fitted line multiplied by $n$ (the number of tubes at each concentration) with the error term of the analysis of variance. The line fitted to the squared data is based on the upper four con-

Table 7. Analysis of variance of Latin square experiment (Table 3)

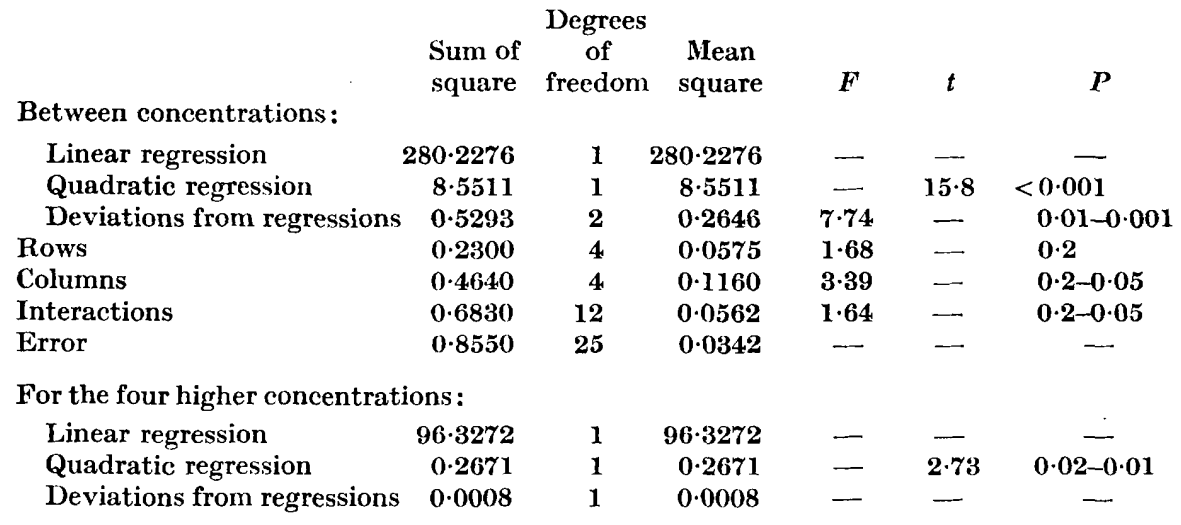

Table 8. Analysis of squared data from Latin square experiment (Table 3)

\begin{tabular}{|c|c|c|c|c|c|c|c|}
\hline 1 & 2 & $\mathbf{3}$ & 4 & 5 & $\boldsymbol{Y}_{w}$ & $W$ & $\boldsymbol{W} \boldsymbol{Y}_{w}$ \\
\hline$x$ & $u$ & $\boldsymbol{Y}$ & $W=4 Y$ & $2 Y(Y-u)$ & $(3-5)$ & - & - \\
\hline 1 & $3 \cdot 63$ & 14.5 & 0.01724 & +1.372 & $13 \cdot 128$ & 0.01724 & $0 \cdot 22633$ \\
\hline 2 & $5 \cdot 31$ & $29 \cdot 3$ & 0.00853 & $+1 \cdot 082$ & $28 \cdot 418$ & 0.01706 & 0.24076 \\
\hline 3 & $6 \cdot 71$ & $44 \cdot 3$ & 0.00564 & -0.666 & 44.966 & 0.01692 & 0.25374 \\
\hline 4 & $7 \cdot 79$ & $59 \cdot 0$ & 0.00424 & $-1 \cdot 690$ & $60 \cdot 690$ & 0.01695 & 0.25714 \\
\hline & & otals & 0.03565 & & & 0.06817 & 0.97797 \\
\hline
\end{tabular}

$$
\begin{aligned}
& n=10 \frac{1}{S(n W)}=0.3565 \quad \bar{x}=1.9125 \quad \bar{y}=27 \cdot 431 \\
& S W(x-\bar{x})^{2}=0.03955 \quad b=15.8599 \\
& S W x(y-\bar{y})=0.62726 \quad y=\bar{y}+b(x-\bar{x}) \\
& =27 \cdot 431+15 \cdot 8599(x-1 \cdot 9125) \\
& n S(u-\sqrt{Y})^{2}=0.053, \quad s^{2}=0.0342, \quad F=1.55, \quad P=>0 \cdot 2 .
\end{aligned}
$$

Variance of estimated value at $x=0$ is

Variance of difference

$$
V\left(Y_{0}\right)=s^{2}\left\{\frac{1}{S(n W)}+\frac{\bar{x}^{2}}{S n W(x-\bar{x})^{2}}\right\}=0 \cdot 0438 .
$$

$$
\bar{y}_{0}-Y_{0}=V\left(y_{0}\right)+V\left(Y_{0}\right)=0.0780, \quad t=10 \cdot 4, \quad P=<0 \cdot 001 .
$$

centrations $(256,64,16$ and $4 \mu \mathrm{g} . / \mathrm{ml}$.), since it is impossible to include the point at $1 \mu \mathrm{g} . / \mathrm{ml}$. in any reasonable provisional line without making the response negative. From Table 6 it will be seen that the observed value departs significantly from the line fitted to the remaining four points. It is therefore assumed that the relation between response and dose is not linear over the whole range. The proper comparison of the linearity of the squared and 
unsquared data is therefore between the upper four concentrations. It will be seen from Table 7 that the unsquared data still depart significantly from linearity when the lowest concentration is omitted, whereas there is no significant departure with the squared data. It is therefore considered preferable to work with the squared data. Furthermore, when using the squared data as described previously for this method, we are justified in assuming the linearity of the relationship in the range 4-256 $\mu \mathrm{g} . / \mathrm{ml}$. At levels below $2 \mu \mathrm{g} . / \mathrm{ml}$. we would tend to overestimate the amount of streptomycin actually present. The assay procedure which has been described employs two control sets of tubes at known concentrations to estimate the strength of a single unknown. This can be analysed using the principles just set out. The weight to be given to the readings at the higher concentrations is less than that attached to the lower as their variance is greater, and, in general, the weight of a mean reading $\bar{y}_{i}$, based on $n_{i}$ tubes is

$$
n_{i} w_{i}=\frac{n_{i}}{4 \bar{y}_{i} s^{2}},
$$

where $s^{2}$ is the 'within concentrations' or error mean square found in the analysis of variance of the unsquared figures. If the mean value of the responses at the two control concentrations are $\bar{y}_{1}$ and $\bar{y}_{2}$, their weighted mean is

$$
\hat{y}=\frac{n_{1} w_{1} y_{1}+n_{2} w_{2} y_{2}}{n_{1} w_{1}+n_{2} w_{2}}
$$

replacing the weights by their values from (7) it will be found that, if $n_{1}=n_{2}$,

$$
\hat{y}=\frac{2}{\left[1 / y_{1}+1 / y_{2}\right]},
$$

the variance of this mean is the reciprocal of the sum of the weights $n_{1} w_{1}$ and $n_{2} w_{2}$, that is

or, in the case where $n_{1}=n_{2}=n$,

$$
\operatorname{Var}(\hat{y})=\frac{4 s^{2}}{\left[n_{1} / \bar{y}_{1}+n_{2} / \bar{y}_{2}\right]},
$$

$$
\operatorname{Var}(\hat{y})=\frac{4 s^{2}}{n\left[1 / y_{1}+1 / \bar{y}_{2}\right]}=\frac{2 s^{2}}{n} \hat{y}
$$

the weight of the unknown reading, $\bar{y}_{3}$, is seen from (7) to be

and its variance is $1 / w_{3}$.

$$
n_{3} w_{3}=\frac{n_{3}}{4 \bar{y}_{3} s^{2}},
$$

The estimate of slope, $b$, is

$$
b=\frac{y_{2}-y_{1}}{x_{2}-x_{1}}
$$

where $x$ is the $\log _{10}$ of the streptomycin concentration, and since $\left(x_{2}-x_{1}\right)$ is fixed by the experimenter,

$$
\begin{aligned}
\operatorname{Var}(b) & =\frac{1}{\left(x_{2}-x_{1}\right)^{2}}\left[\operatorname{Var}\left(\bar{y}_{1}\right)+\operatorname{Var}\left(\bar{y}_{2}\right)\right] \\
& =\frac{4 s^{2}}{\left(x_{2}-x_{1}\right)^{2}}\left[\frac{\bar{y}_{1}}{n_{1}}+\frac{\bar{y}_{2}}{n_{2}}\right],
\end{aligned}
$$


the variances of $\bar{y}_{1}$ and $\bar{y}_{2}$ being found as before from (7). When $n_{1}=n_{2}=n$

$$
\operatorname{Var}(b)=\frac{4 s^{2}\left(\bar{y}_{2}+\bar{y}_{1}\right)}{n\left(x_{2}-x_{1}\right)^{2}}
$$

to find the concentration of the unknown solution, $x_{3}$, we have

$$
x_{3}=\frac{\bar{y}_{3}-\hat{y}}{b}+\hat{x}
$$

where $\hat{x}$ is the weighted mean of the $\log$ concentrations of the standard preparation.

In the general case where the numbers of tubes at the two dose levels are unequal, $n_{1}$ and $n_{2}$ say, we have

$$
\hat{x}=\left[\frac{n_{1} x_{1}}{\bar{y}_{1}}+\frac{n_{2} x_{2}}{\bar{y}_{2}}\right] /\left[\frac{n_{1}}{y_{1}}+\frac{n_{2}}{y_{2}}\right],
$$

so when $n_{1}=n_{2} \quad \hat{x}=\frac{x_{1}}{\bar{y}_{1}}+\frac{x_{2}}{\bar{y}_{2}} / \frac{1}{\bar{y}_{1}}+\frac{1}{\bar{y}_{2}}$.

As an example of this process we may take the experimental results from Table 1:

\begin{tabular}{lccl} 
& $x$ & $n$ & \multicolumn{1}{c}{ Mean of squared readings } \\
Control at $4 \mu \mathrm{g} . / \mathrm{ml}$. & $0 \cdot 60206$ & 6 & $13 \cdot 0983) S^{2}=0 \cdot 0222$ \\
Control at $64 \mu \mathrm{g} . / \mathrm{ml}$. & 1.80618 & 6 & $50 \cdot 9033\} 10$ degrees of freedom \\
Unknown & & 4 & $\mathbf{2 9 \cdot 4 4 2 5}$
\end{tabular}

then

$$
b=\frac{50 \cdot 9033-13 \cdot 0983}{1 \cdot 80618-0 \cdot 60206}=31 \cdot 3964
$$

and

$$
\begin{aligned}
\hat{x} & =\frac{1 \cdot 80618}{50 \cdot 9033}+\frac{0 \cdot 60206}{13 \cdot 0983} / \frac{1}{50 \cdot 9033}+\frac{1}{13 \cdot 0983} \\
& =0 \cdot 8483,
\end{aligned}
$$

and

$$
\begin{aligned}
\hat{y} & =\frac{2}{\frac{1}{50 \cdot 9033}+\frac{1}{13 \cdot 0983}} \\
& =20 \cdot 8158,
\end{aligned}
$$

so that, from (13), we estimate

$$
\begin{aligned}
x_{3} & =\frac{29 \cdot 4425-20 \cdot 8158}{31 \cdot 3964}+0 \cdot 8483 \\
& =1 \cdot 1231 .
\end{aligned}
$$

We have now to find the variance of $x_{3}$. This consists of two parts, one but not the other involving the standard error of $b$, thus

$$
\operatorname{Var}\left(x_{3}\right)=\frac{\operatorname{Var}(\hat{y})+\operatorname{Var}\left(\bar{y}_{3}\right)}{b^{2}}+\frac{\left(\bar{y}_{1}-\bar{y}_{2}\right)^{2} \sigma_{b}^{2}}{b^{4}}
$$

where $\sigma_{b}^{2}$ is the variance of $b$, given in equation (12), and is the variance of the difference between $\bar{y}_{3}$ and $\hat{y}$, which is the sum of the variances of $\bar{y}_{3}$ and $\hat{y}$, given by equations (11) and $(11 a)$; so that since $n_{1}=n_{2}$

$$
\begin{aligned}
\operatorname{Var}(\hat{y}) & =\frac{0.0222 \times 2 \times 20.8158}{6} \\
& =0.1540,
\end{aligned}
$$


and

$$
\begin{aligned}
\operatorname{Var}\left(\bar{y}_{3}\right) & =\frac{4 \times 29 \cdot 4425 \times 0.0222}{4} \\
& =0.6536 .
\end{aligned}
$$

So

$$
\begin{aligned}
\operatorname{Var}(\hat{y})+\operatorname{Var}\left(\bar{y}_{3}\right) & =0 \cdot 1540+0.6536 \\
& =0.8076 .
\end{aligned}
$$

now

$$
\operatorname{Var}(b)=\frac{4 \times 0.0222 \times 64.0016}{8.6995}
$$

$$
=0.6553 \text { from }(12 a) \text {, }
$$

so we can now calculate

$$
\begin{aligned}
\sigma_{x_{3}}^{2} & =\frac{0.8076}{31 \cdot 3964}+\frac{(50.9033-13 \cdot 0983)^{2} \times 0.6533}{971,672} \\
& =0.000819+0.000961 \\
& =0.00178
\end{aligned}
$$

and we have finally

$$
x_{3}=1 \cdot 1231 \pm 0 \cdot 0422 \text {. }
$$

Using the value of Student's $t$ for the $5 \%$ point on 10 degrees of freedom we find the limits of error of $M$ as

$$
1 \cdot 0291-1 \cdot 2216
$$

giving an experimental error of about $8 \%$ in logarithmic units and of $+27 \%$ and $-19 \%$ in units of concentration. These limits are, of course, calculated for one particular experiment and can only be considered as typical of those likely to be found in other similar experiments. In a routine assay the fluids composing the controls and unknown are different and, as previously indicated, an increased estimate of the error must therefore be made.

A further statistical point which deserves to be mentioned is the very high degree of grouping which the experimental method imposes on the data. The readings are made to the nearest $0.1 \mathrm{~mm}$. That is to say, the grouping unit is $0.1 \mathrm{~mm}$., which is of about the same order of magnitude as the error standard deviation of the technique. For this reason it would be as well to have at least 5 degrees of freedom for the estimate of the error mean square. Sheppard's corrections should not be used when making tests of significance on the data.

\section{REFERENCES}

Brownlee, K. A., Delves, C. S., Dorman, M., Green, C. A., Grenfell, F., Johnson, J. D. A. \& Smrth, N. (1948). The biological assay of streptomycin by a modified cylinder plate method. J. gen. Microbiol. 2, 40.

Challinor, S. W. \& King, H. K. (1947). Production of streptomycin. Edinb. med. J. 54, 465-75.

Cooper, K. E. \& Woodman, D. (1946). Diffusion of antiseptics through agar gels. J. Path. Bact. 58, 75-84.

Finney, D. J. (1944). Mathematics of biological assay. Nature, Lond., 153, 284.

Finney, D. J. (1947a). Probit Analysis. Cambridge University Press.

Finney, D. J. (1947b). Principles of biological assay. Suppl. J. R. statist. Soc. 9, 46-81.

Geiger, W. N., Green, S. R. \& Waksman, S. A. (1946). Inactivation of streptomycin and its practical applications. Proc. Soc. exp. Biol., N.Y., 61, 187-92. 
HAYES, W. (1945). Effect of agar depth in the plate method for the assay of penicillin. J. Path. Bact. 57, 457.

Konnegay, G. B., Forgacs, J. \& Henley, T. (1946). Studies on streptomycin. II. Blood levels and urinary excretion in man and animals. J. lab. clin. Med. 31, 523-34.

Lenher, S. \& Smith, J. E. (1936). A diffusion study of dyes. J. phys. Chem. 40, 1005 .

Levy, A. \& Roth, L. (1936). Elements of probability, p. 125. Oxford: Clarendon Press.

Loo, Y. H., McGuire, J. M., Savage, G. M., Skell, P. S., Thornberry, H. H., Erlich, J. \& Sylvester, J. C. (1945). Assay of streptomycin by paper disk method. J. Bact. 50, 701-9.

May, J. R., Voureka, A. E. \& Fleming, A. (1947). Some problems in the titration of streptomycin. Brit. med. J. i, 627-30.

Mellor, J. W. (1912). Higher Mathematics for Students of Chemistry and Physics, 2nd ed., p. 481. London: Longmans, Green \& Co.

Stebbins, R. B. \& Robinson, H. J. (1945). Method for determination of streptomycin in body fluids. Proc. Soc. exp. Biol., N.Y., 59, 225-57.

Vesterdal, J. (1947). Studies on the inhibition zones observed in the agar cup method for penicillin assay. Acta path. microbiol. Scand. 24, 272.

Waksman, S. A., Bugie, E. \& Schatz, A. (1944). Streptomycin, a substance exhibiting antibiotic activity against gram-positive and gram-negative bacteria. Proc. Soc. exp. Biol., N.Y., 55, 66-9.

(Received 8 July 1948) 